\title{
eddy: Extracting Protoplanetary Disk Dynamics with Python
}

\section{Richard Teague ${ }^{1}$}

1 University of Michigan

DOI: $10.21105 /$ joss. 01220

\section{Software}

- Review 七

- Repository ca

- Archive ca

Submitted: 22 January 2019 Published: 26 February 2019

\section{License}

Authors of papers retain copyright and release the work under a Creative Commons Attribution 4.0 International License (CC-BY).

\section{Summary}

eddy is a Python package which implements several methods for extracting kinematical information from astronomical observations of Doppler shifted molecular line emission in protoplanetary disks, the birthplace of planets. Such kinematic information is essential for constraining the physical structure of the accretion disk and searching for evidence of embedded planets (Teague, Bae, Bergin, Birnstiel, \& Foreman-Mackey, 2018a; Teague, Bae, Birnstiel, \& Bergin, 2018b). Basic functionality includes two types of analysis: either for full 2D rotation maps ("first moment maps"), or for a 1D annulus of spectra extracted from a particular radius in the disk.

Fitting a first-moment map is a frequently used analysis in the study of protoplanetary disks, typically used to constrain the mass of the central star. As eddy includes the ability to have a 3D geometry, an increasingly more important consideration when modelling high spatial resolution observations, this quickly allows the user to constrain basic disk geometrical properties and rotation direction of the disk. A simple dictionary interface allows the user to vary specific parameters in the fitting while holding values constrained from other observations constant.

eddy also implements the methods described in (Teague et al., 2018a) and (Teague et al., 2018 b) to infer the rotation velocity based on the relative shift of spectra from different regions in the disk. The precision achieved with this method makes it possible to infer local deviations in the rotation velocity due to the presence of an embedded planet.

There are several Jupyter Notebooks of examples of how to use of the the specific functions as well as some test data for the user. The functions within eddy.modelling allows the user to generate synthetic spectra for testing the methods and designing observations. These functions were used in the generation of the Appendix in (Teague et al., 2018b).

\section{Acknowledgements}

We acknowledge Dan Foreman-Mackey for helping with the development of the implementation and Til Birnstiel for the development of the documentation.

\section{References}

Teague, R., Bae, J., Bergin, E. A., Birnstiel, T., \& Foreman-Mackey, D. (2018a). A Kinematical Detection of Two Embedded Jupiter-mass Planets in HD 163296, 860, L12. doi:10.3847/2041-8213/aac6d7

Teague, (2019). eddy: Extracting Protoplanetary Disk Dynamics with Python. Journal of Open Source Software, 4(34), 1220. https: 1 
Teague, R., Bae, J., Birnstiel, T., \& Bergin, E. A. (2018b). Evidence for a Vertical Dependence on the Pressure Structure in AS 209, 868, 113. doi:10.3847/1538-4357/aae836 